\title{
Research of Scenario Inference of Emergency Based on OWL and SWRL
}

\author{
Yu Liu*, Shihong Chen, Lei Chen and Yunhua Wang \\ School of Computer Science and Technology, Wuhan University of Science and Technology, Wuhan 430065, China
}

\begin{abstract}
Although the scenario analysis has been extensively sudied in the area of emergency managnemt, the heterogeneous data and the lack of semantic meaning always hamper the performance of scenario inference in many emergency response systems. Aiming at this problem, we proposed a scenario inference model and implemented this model with Web Ontology Language (OWL) and Semantic Web Rule Language (SWRL). Furthermore, an algorithm of scenario inference was designed to assist the decision-makers to find the potential hazard scenarios. In order to testify the validity of scenario inference model, we employed some inference rules drawn from the historical emergencies to deduce the east Japan earthquake. The experimental result shows that our method is effective for emergency response system.
\end{abstract}

Keywords: Emergency Response System, OWL, Scenario Inference, Scenario Ontology, SWRL.

\section{INTRODUCTION}

An emergency is an unexpected and dangerous situation, which happens suddenly and requires the quick response to deal with it. In order to help people to solve various issues in emergency, many emergency response systems based on information technologies have been designed and implemented. As one of core technologies of emergency response system, the real-time scenario analysis is the indispensable module in these systems and can provide the basic information for scientific decision-making. The scenario inference is a hotspot of scenario analysis research, and some achievements have been gained in recent years. On the basis of PSR model and Bayesian networks, Yuan et al. had done some researches on the scenario inference model to meet the requirements of emergency decision-making [1]. According to the periodic characteristics and constraints of emergencies, the paper [2] proposed a formal expression method of scenario ontology and a scenario model with three levels (such as factor, state and event), and then analyzed the evolution relationships between and in these levels to design a description method of scenario evolution. Although some progresses have been achieved, there are some inevitable problems that all emergency response systems must face. For example, the heterogeneous data and the lack of semantic meaning may hinder the performance of scenario inference in these systems.

Aiming at the problem above, more and more researchers had tended to employ the semantic technology to obtain the solution [3-5]. Recently, some semantic technologies had been applied to express knowledge in emergencies [6-8], integrate data from different sources [4], and model the formal plan for emergency management [9], but there are few researches on taking the advantage of the reasoning power of semantic web to infer the scenario evolution in emergencies. Although Li et al. had used SWRL [10] to analyze the emergency evolution, and the event chain model proposed by them cannot effectively describe the intricate relationships between scenarios in emergencies [11]. In order to make up for the above deficiencies, some works that we had done are listed in brief as the followings. First, we proposed the architecture of emergency response system and designed the evolution model in the level of scenario inference, which can describe the complex relations between scenarios. Second, the scenario ontology for the architecture was implemented with OWL [12]. Third, we employed SWRL to define the rules of scenario inference on the basis of the core ontology of emergency, and then provided the algorithm of scenario inference to find the potential hazards. Finally, we verified the effectiveness of our method by analyzing the earthquake happened in east Japan.

\section{ARCHITECTURE OF EMERGENCY RESPONSE SYSTEM BASED ON SCENARIO}

In this paper, the architecture of emergency response system based on scenario consists of four modules: data source, data processing, scenario inference and decision supporting. Fig. (1) is showing the relations between these modules and some sub modules in them. To ensure the extensibility of this architecture, the data source layer contains several frequently-used data sets that have been employed in some emergency response systems, such as RDBMS, linked open data [13], social network and mobile equipment. As soon as the data processing layer retrieves data from various data sources, some operations for data transformation and identity resolution will be executed to solve the schema differences between these data sources. Then, the sub module of data fusion evaluates the credibility of various data and deal with 


\begin{tabular}{|l|c|c|}
\hline Decision & Responsing & Resource \\
\hline Supporting & Plans & Scheduling \\
\hline
\end{tabular}
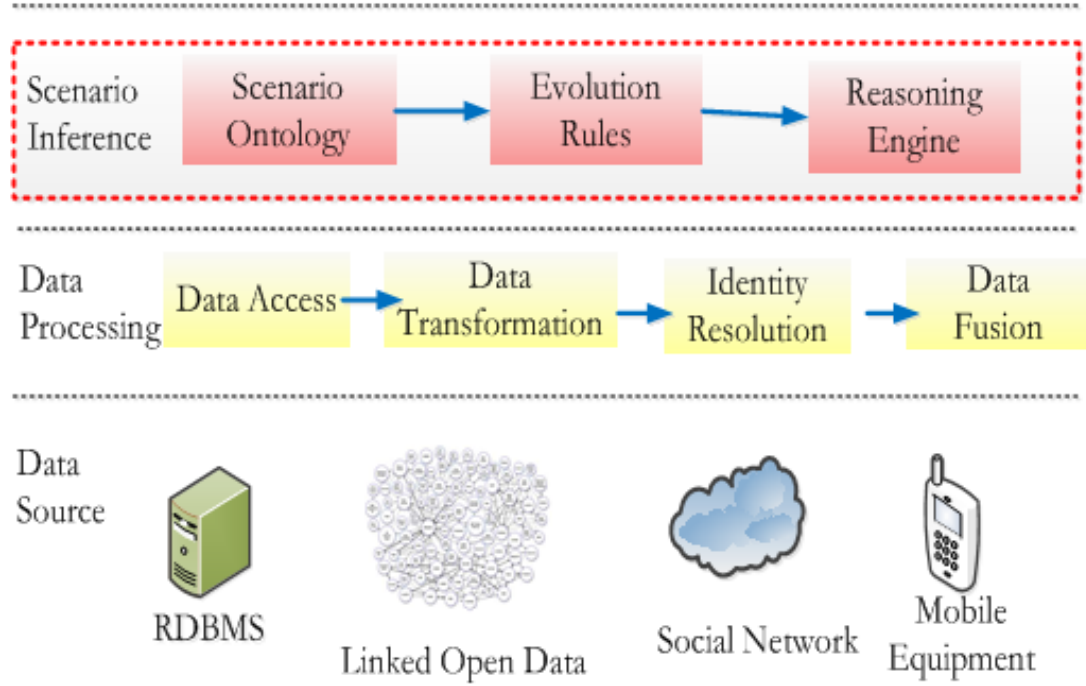

Fig. (1). Architecture of emergency response system based on scenario.

the data collisions so that it can provide some reliable data to the scenario inference layer. On the basis of scenario ontology, evolution rules and the underlying data, the reasoning engine makes a deduction to find the potential scenarios that may come out in the future. Moreover, the scenario inference layer should provide some query interfaces for the decision supporting layer. Once these potential scenarios are queried upon, some sub modules in the decision supporting layer are triggered to provide a number of rational proposals for the decision-maker on the basis of responding plans, which are prepared in advance. For example, the sub module of resource scheduling can help people to allocate the limited resources scientifically. The highlight of this paper is the level of scenario inference, which is surrounded by a dotted box in Fig. (1).

\section{SCENARIO EVOLUTION MODEL}

Due to the complexity of internal and external factors, some emergencies usually derive a variety of hazard factors, the damage of which may be greater than the initial one. Here, we firstly give the formal definitions of scenario, scenario evolution and event that are the basis of model of scenario evolution.

As far as scenario concerned, there are many different definitions about it. Kahn regards scenarios as a series of facts from the initial state to some certain states in the future [14]. In [15], scenarios are the comprehensive descriptions of possible events in the future. In this paper, we consider that scenarios are factors drawn from history events, just as the definitions listed as the following.
Definition 1 Scenario is a certain factor that exists in the history events or the evolution rules. For example, earthquake, tsunami, unclear leakage and loss of life all are regarded as scenarios. In the later of this paper, a scenario and a set of scenario are denoted as s and $\mathrm{S}$ respectively.

Definition 2 Scenario Attribute is used to describe the feature of scenario, which always includes multiple scenario attributes. For example, the features of earthquake contain magnitude, type and position, and so on. A(s) stands for the attribute set of a scenario s.

Definition 3 Scenario Evolution Rule describes the transformation relation between scenarios. Generally, a scenario evolution rule can be regarded as a function, so that the input is the set of causing scenario and the output is the set of result scenario.

Definition 4 Event is the description of a series of scenarios. For example, the earthquake happened in the east of Japan is an event, which contains structural damage, tsunami, equipment damage and unclear leakage sequentially.

On the basis of definitions above, the model of scenario evolution can be constructed by drawing the relations between different scenarios from some historical events and known rules of scenario evolution. Fig. (2) explains the procedure of model construction and the model structure of scenario evolution, which is comprised of scenarios and the relations between them. Each scenario in the model always has several attributes, and the directed edges between scenarios stand for the cause and effect between them. 


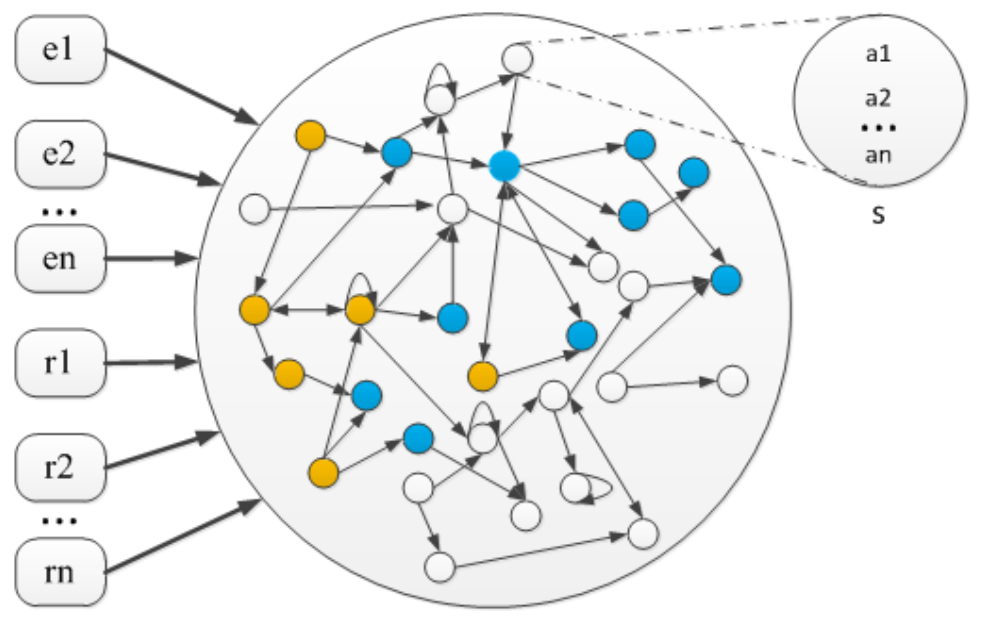

Fig. (2). Scenario evolution model drawn from events and rules.

\section{THE IMPLEMETATION OF SCENARIO EVOLU- TION MODEL}

Just as the first section introduced, semantic technologies had been widely used in the construction of emergency response system. In order to implement the scenario evaluation model, we followed this idea and take advantages of OWL and SWRL to construct the model. First of all, the core-level scenario ontology of emergency is built with OWL so that the scenario ontology for different domains can be constructed by extending this core-level ontology. To make up the lack of expression power of OWL, SWRL is employed in the implementation of scenario evolution rule. At the end of this section, an algorithm of scenario inference was designed to find the potential hazard scenarios.

\subsection{The Core-level Scenario Ontology}

Considering PROTON [16] providing the coverage of general concepts necessary for a wide range of tasks, we implemented the core-level scenario ontology by extending PROTON. The core-level scenario ontology includes three modules: event module, scenario module and mitigation module. The event module describes the emergency classification and some basic attributes. In the most studies, emergency can be divided into four types: natural disaster, accident disaster, public health event and social security event. The scenario module defines a basic class, "Scenario", which has three subclasses. Among them, "Context" is the class of environment information, such as weather, topography and social situation; the instances of "HazardFactor" may endanger people, society or natural resource; "AffectedObject" includes objects that are affected by some certain hazard factors. The mitigation module contains some classes that will be used for the decision supporting. Fig. (3) shows the core-level scenario ontology in detail.

\subsection{SWRL-based Scenario Evolution Rule}

Although the OWL-based scenario ontology can use some axioms to infer some potential scenarios from existing scenarios, it is limited by the expression ability of OWL and cannot describe all relations between scenarios. Aiming at this problem, SWRL is adopted to define the scenario evolution rules in the level of scenario inference. Because SWRL combines OWL with RuleML, it can integrate the OWLbased ontology with rules seamlessly. Consequently, SWRLbased scenario evolution rules not only can interact with the scenario information described with OWL, but also can get maximum reuses from it. A SWRL rule is comprised of antecedent and consequent, both of which consist of zero and more atoms. Hence, a SWRL rule implies that the conditions specified in the consequent must be true if all atoms in the antecedent are satisfied. Moreover, SWRL also provide a set of built-ins that can be used to judge the relations between objects of various types, such as String, Boolean, URIs, and so on. All features of SWRL introduced above can benefit the construction of scenario evolution rules.

Formula (1), (2), (3) are three SWRL-based scenario evolution rules about the domain of earthquake. The first rule describes that a building may be damaged if the building is in the effect area of earthquake and the anti-seismic level of the building is less than the magnitude of the earthquake. In formula (2), the rule of scenario evolution is that some hazardous goods may leak if the hazardous goods are stored in the damaged

Building which someone may be harmed if he is in the expansion area of the dangerous goods, shown as formula (3). It is noteworthy that "InAreaOf" is custom built-ins of SWRL for the domain of earthquake.

Earthquake(?e)^HasMagnitude(?e,?em $)^{\wedge}$
Building(?b) ${ }^{\wedge}$ HasAntiseismic(?b,?ba $)^{\wedge}$
InAreaOf(?b,?e $)^{\wedge}$ swrlb:lessThan(?ba,?em $)^{\wedge}$
->DamagedBuilding(?b)

DamagedBuilding(?db) ${ }^{\wedge}$ HazardousGoods $(? h g)^{\wedge}$

Storage(?hg,?db)->HazardousGoodsLeak(?hg) 


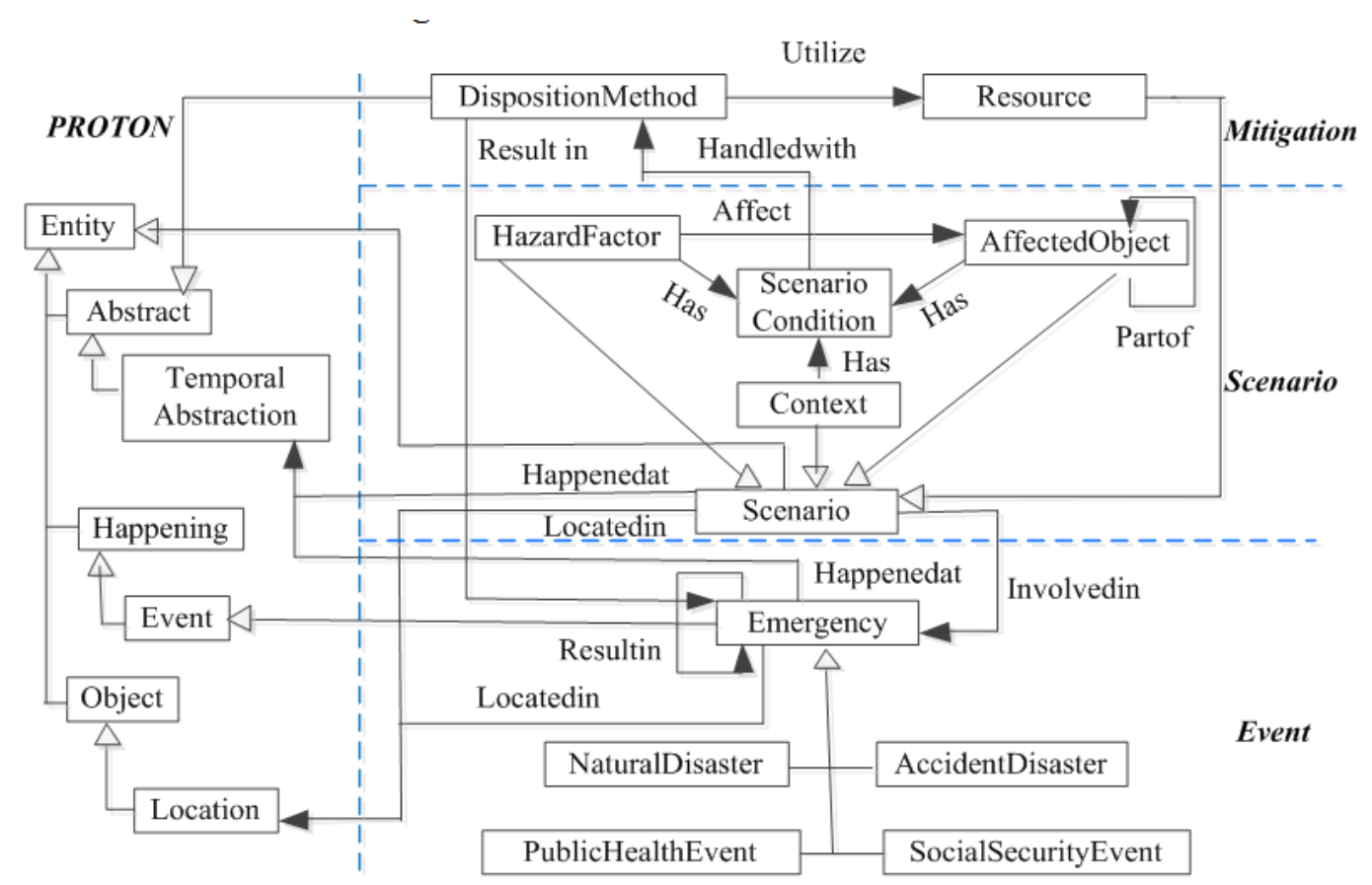

Fig. (3). The core-level scenario ontology.

HazardousGoodsLeak(?hg $)^{\wedge}$ Person(?p $)^{\wedge}$

Person(?p) ${ }^{\wedge}$ InAreaOf(?p, ?hg) $\rightarrow$ InjuredPerson(?p)

\subsection{Algorithm of Scenario Inference}

Owing to the complexity and derivation of emergency, it is impossible to obtain enough information to deduce out the potential scenarios, especially at the initial phase. Besides that, the performance of scenario inference will be reduced if all scenario evolution rules are imports into the scenario evolution model. To solve these problems, we proposed an algorithm of scenario inference to assist the decision-makers to find the potential hazard scenarios quickly. According to the scenario instances and evolution rules that are provided by the user, the algorithm can keep inferring until the evolution is terminated. Furthermore, user can add some new scenarios and rules during the execution of algorithm. The detail of algorithm is listed as the following:

Algorithm ASR

Input: a set of scenario instance (siSet), a set of scenario evolution rule (serSet).

Output: a set of potential hazard scenario (phsSet)

1: initialize a scenario evolution model, seModel

2: while (evolution is not terminated)

3: lock siSet, lock serSet

4: for each $\mathrm{x}$ in siSet

5: import $\mathrm{x}$ into seModel
6: remove $\mathrm{x}$ from siSet

7: find rSet that includes $\mathrm{x}$ in the antecedent

8: append serSet with rSet

9: end for

10: for each $r$ in serSet

11: import $r$ into seModel

12: remove $r$ from serSet

13: end for

14: unlock siSet, unlock serSet

15: hasNew $=$ true

16: while $($ hasNew $==$ true $)$

17: execute the inference on seModel

18: if (seModel has some new scenarios)

19: find nrSet that includes new scenarios

20: import nrSet into seModel

21: else hasNew $=$ false

22: end while

23: end while

24: find all potential hazard scenarios in seModel and insert them into phsSet

25: return phsSet 
Table 1. Some emergencies in history.

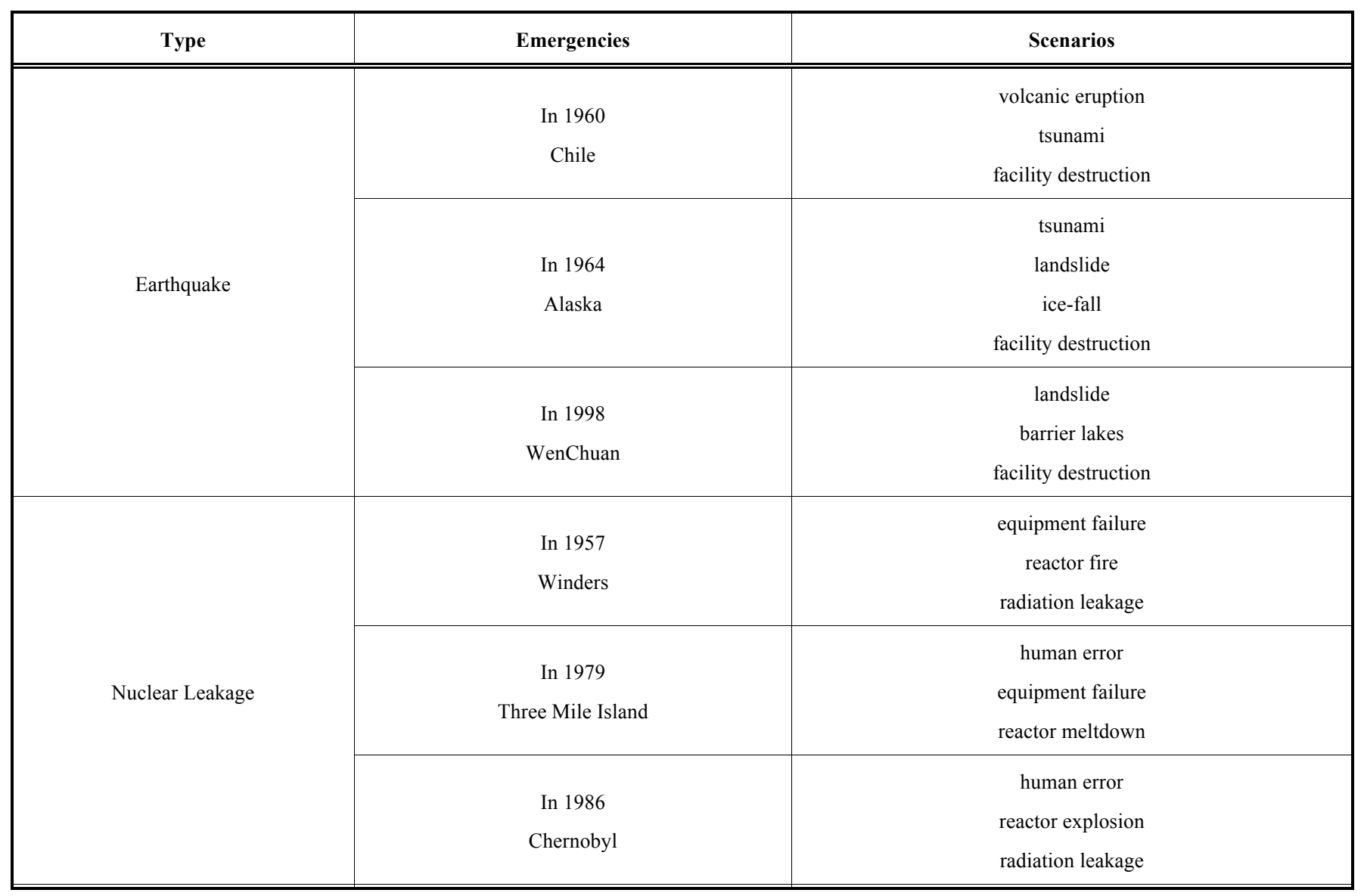

\section{EXMAPLE OF SCENARIO INFERENCE}

In this section, the scenario ontology and evolution rules are drawn from some historical emergencies, and then are used to deduce the earthquake that happened at the east area of Japan in 2011.

Since the earthquake and nuclear leakage were involved in the east Japan earthquake, the emergencies that we referred to should include some historical emergencies of these two types. Table 1 lists some emergencies that had great influence in the $20^{\text {th }}$ Century. Obviously, the earthquakes share some same scenarios with the nuclear leakages, such as facility destruction and equipment failure. In addition, each emergency has some specific characteristics. For example, the earthquakes happened in Chile and Alaska both caused tsunami because the locations of earthquakes are nearby the ocean, the barrier lakes were formed by the landslides and rivers in the earthquake of WenChuan, the reactor cached fire in the nuclear leakage of Winders. By analyzing these common and specific characteristics, we constructed the domain ontologies of earthquake and nuclear leakage with OWL by extending the core-level scenario ontology introduced above.

According to the emergencies listed in Table 1, we conclude some scenario evolution rules. The following content demonstrates part of these rules. Formula (4) and (5) can reason out the barrier lakes when some mountains and rivers exist in the effect area of earthquake, just as the scenarios happened in the WenChuan earthquake. In the same way, the scenarios of tsunami and nuclear leakage can be achieved by inferring the formula (6-9).

Earthquake(?e) $)^{\wedge}$ HasMagnitude(?e,?em $)^{\wedge}$

Mountain $(? \mathrm{~m})^{\wedge} \operatorname{InAreaOf}(? \mathrm{~m}, ? \mathrm{e})^{\wedge}$

swrlb : greaterThan(?em,8)^ - > Landslide(?m)

River(?r $)^{\wedge}$ Landsllide(?1)^ Nearby(?m,?r)

- > BarrierLake(?r)

Earthquake(?e)^Sea(?s)^ InAreaOf(?s, ?e $)^{\wedge}$

HasMagnitude(?e,?em)^swrlb : greaterThan(?em, 8$)^{\wedge}$

- > Tsunami(?s)

City(?c $)^{\wedge}$ Tsunami(?t)^ Nearby(?c,?t)

- > CityDamaged(?c)

Earthquake(?e)^ Facility(?f)^ InAreaOf(?f,?e)

- > FacilityDamaged(?f) 


$$
\begin{aligned}
& \text { FacilityDamaged(?f)^ NuclearPowerStation(?n })^{\wedge} \\
& \text { KeyPartOf(?f,?n)- > NuclerLeakage(?n) }
\end{aligned}
$$

In order to testify the effectiveness of our method, the scenario ontologies and rules drawn from the historical emergencies were used to deduce the earthquake of east Japan. Before the task of scenario inference, some instances in the effect area of the earthquake were prepared and imported into the scenario evolution model. Then, Pellet [17] and the algorithm of scenario inference were used to reason out the potential scenarios. The experimental results show that the scenario inference based on OWL and SWRL can help the decision-maker find some potential hazards quickly, even at the early stage of emergency. Consequently, some disaster preventions and reductions can be done ahead of time to decrease losses in the emergency.

\section{CONCLUSION AND FUTURE WORKS}

In this paper, we researched the scenario inference of emergency based on OWL and SWRL. By taking advantage of the reasoning power of OWL and SWRL, our method can help the decision-maker find the potential hazards in advance. In the future, we will follow two directions to conduct our researches. One is the application of uncertain reasoning technology in the scenario inference; the other is the application of semantic reasoning technology in the process of decision making.

\section{CONFLICT OF INTEREST}

The authors confirm that this article content has no conflict of interest.

\section{ACKNOWLEDGEMENTS}

This work was partly supported by the Science Guidance Project of Education Department of Hubei Province under Grant No. B2014085, Science Foundation for Yong Teachers of Wuhan University of Science and Technology under Grant No. 2012XZ015.

\section{REFERENCES}

X.F. Yuan, S.C. Tian, and L. Wang, "Scenario analysis of unconventional emergency based on PSR model and bayeian networks,"
Chinese Journal of Safety Science, vol. 21, no. 1, pp. 169-176, 2011.

[2] Y.X. Wang, Research on Context Reconstruction Model of Unconventional Emergency, Harbin Industrial Unversity, 2011, http://www.dissertationtopic.net/doc/1480170

[3] T. Silva, V. Wuwongse, and H.N. Sharma, "Disaster mitigation and preparedness using linked open data," Journal of Ambient Intelligence and Humanized Computing, vol. 4, no. 5, pp. 591-602, 2012

[4] G. Babitski, S. Bergweiler, O. Grebner, D. Oberle, H. Paulheim, and F. Probst, "SoKNOS - Using Semantic Technologies in Disaster Management Software," The Semantic Web: Research and Applications Lecture Notes in Computer Science, vol. 6644, pp. 183197, 2011.

[5] C. De Maio, G. Fenza, M. Gaeta, V. Loia, and F. Orciuoli, "A knowledge-based framework for emergency DSS," Knowledge Based System, vol. 24, no. 8, pp. 1372-1379, 2011.

[6] Y. Liu, S.H. Chen, and Y.H. Wang, "SOFERS: scenario ontology for emergency response system," Journal of Networks, vol. 9, no. 9, pp. 2529-2535, 2014.

[7] H.Z. Wang, and X.M. Mao, "Knowledge representation based on owl for emergency response in the field of natural disasters," Computer Systems and Applications, vol. 21, no. 4, pp. 207-211, 2012.

[8] Y.H. Yang, J.P. Du, and M.Y. Liang, "Emergency domain knowledge modeling based on ontolgoy," Journal of Contral South University (Science and Technology), vol. 42, no. 1, pp. 859-864, 2011.

[9] J.D. Yang, Study on Semantic Model of digital Emergency Plan Under the Scenarios-Cope Mode, Nanjing University of pots and telecommunications, 2012.

[10] SWRL: A Semantic Web Rule Language Combinng OWL and RuleML. W3C: http://www.w3.org/Submission/SWRL/, 2004

[11] C.D. Li, T. Xie, J. Li, and H.X. Hong. "Knowledge expression and chained evolution reasoning of emergencies based on SWRL", Journal of Information, vol. 31, no. 5, pp. 55-61, 2012

[12] OWL Web Ontology Language Overview. W3C: http://www.w3.org/TR/owl-features/, 2009

[13] M. Hausenblas, "Understanding Linked Open Data as a Web-Scale Database", In: International Conferrence on Advances in Databases Knowledge and Data Applications, 2010, pp. 56-61.

[14] H. Kahn, and A. Wiener, The Year 2000:A Framework for Speculation on the Next Thirty-three Years. MacMillan: New York, 1967.

[15] W. Ian, "From scenario thinking to strategic action," Technological Forecasting and Social Change, vol. 65, no. 1, pp. 23-29, 2000.

[16] I. Terziev, A. Kiryakov, and D. Manov, "Base Upper-level Ontology (BULO) Guidance Deliverable 1.8.1", SEKT project, 2005.

[17] E. Sirin, B. Parsia, B.C. Grau, A. Kalyanpur, and Y. Katz "Pellet: A practical owl-dl reasoner," Web Semantics: Science, Services and Agents on the World Wide Web, vol. 5, no. 2, pp. 51-53, 2007.

(C) Liu et al.; Licensee Bentham Open.

This is an open access article licensed under the terms of the Creative Commons Attribution Non-Commercial License (http://creativecommons.org/licenses/by-nc/3.0/) which permits unrestricted, non-commercial use, distribution and reproduction in any medium, provided the work is properly cited. 